\title{
U-Shaped Association of Body Mass Index with the Risk of Peripheral Arterial Disease in Chinese Hypertensive Population
}

\author{
Junpei $\mathrm{Li}^{1}$ \\ Shichao $\mathrm{Yu}^{2}$ \\ Wei Zhou ${ }^{1,3}$ \\ Linjuan Zhu ${ }^{1,3}$ \\ Tao Wang ${ }^{1,3}$ \\ Huihui Baol,3 \\ Xiao Huang $\mathbb{D}^{\prime}$ \\ Xiaoshu Cheng ${ }^{1,3}$ \\ 'Department of Cardiovascular, the \\ Second Affiliated Hospital of Nanchang \\ University, Nanchang of Jiangxi, People's \\ Republic of China; ${ }^{2}$ Qinghua Health \\ Center, Wuyuan, People's Republic of \\ China; ${ }^{3}$ Center for Prevention and \\ Treatment of Cardiovascular Diseases, \\ the Second Affiliated Hospital of \\ Nanchang University, Nanchang of Jiangxi, \\ People's Republic of China
}

Background: High body mass index (BMI) is a well-recognized risk factor for cardiovascular diseases. But its role in peripheral artery disease (PAD) remains perplexing. Our study aims to evaluate the association of BMI with PAD in the Chinese hypertensive population. Methods: This is a cross-sectional study with enrollment data from the Chinese H-type Hypertension Registry.10896 hypertensive patients aged $\geq 18$ years were included in the final analysis.

Results: The prevalence of PAD diagnosed by ABI in this study was $3.2 \% \quad(n=351)$. A U-shaped association between BMI and PAD was found. Per SD increment $\left(3.6 \mathrm{~kg} / \mathrm{m}^{2}\right)$ on the left side of the BMI threshold (BMI $<25.7 \mathrm{~kg} / \mathrm{m}^{2}$ ) was associated with a $27 \%$ decrease in the adjusted risk of PAD [OR, 0.73; 95\% confidence interval (CI) 0.60, 0.89; $\mathrm{P}=0.002]$; BMI was significantly positively associated with the risk of PAD (OR, 1.52; 95\% CI 1.52, 1.93; $\mathrm{P}=0.001$ ) in those with $\mathrm{BMI} \geq 25.7 \mathrm{~kg} / \mathrm{m}^{2}$.

Conclusion: In summary, a U-shaped association between BMI and the risk of PAD in the Chinese hypertensive population was found. BMI with the lowest risk of PAD was estimated to be $25.7 \mathrm{~kg} / \mathrm{m}^{2}$.

Keywords: peripheral arterial disease, body mass index, hypertension

\section{Introduction}

Peripheral arterial disease (PAD) is the third leading atherosclerotic disease after coronary heart disease and stroke, ${ }^{1}$ mainly caused by the accumulation of lipid and fibrous material between the intima and media of lower limb arteries, resulting in luminal stenosis (focal or diffuse). It is well known for a sharp increase in the prevalence of PAD with advanced age. $^{2,3}$ With the aging of the Chinese population, PAD has become an increasingly severe clinical and social problem. Allison et al also showed ethnic differences were independent factors in the prevalence of PAD. ${ }^{4}$ Compared to Whites, Blacks seem to be more vulnerable to PAD, while Asians seem to have a lower prevalence of PAD. ${ }^{5}$

The prevalence of PAD was higher in people with underweight, but the association between BMI and PAD was uncertain due to a variety of potential covariates. ${ }^{6,7}$ A small prospective cohort study showed that obesity independently predicts severe PAD. ${ }^{8}$ However, the recent observational study with more than 3 million sample size has found J-shaped relationship between BMI and PAD only in females. ${ }^{9}$ Epidemiology of Dementia in Central Africa (EPIDEMCA) study recruited the elderly in the Central African Republic and the Republic of Congo, showed underweight and obesity were all associated with the risk of PAD. ${ }^{10}$ 
Due to the inconsistent and the evidence of association between $\mathrm{BMI}$ and prevalence of PAD in the Chinese was still lacked. Our study aims to explore the association between BMI and the risk of PAD in Chinese hypertensive patients.

\section{Methods}

\section{Study Design and Participants}

The study population was drawn from the China Hypertension Registry (Registration number: ChiCTR1800017274), a real-world observational registry of hypertension designed to investigate the prevalence and treatment of hypertension in China and to assess prognostic risk factors. Details of the inclusion and exclusion criteria for the study have been published. ${ }^{11}$ From March 2018 to August 2018, we recruited a total of 14,268 study participants in Wuyuan, Jiangxi Province, China as our study population, and finally analyzed the data of 10,802 .

\section{Ethics Statement}

The study was conducted in accordance with the Declaration of Helsinki and approved by the Ethics Committee of the Institute of Biomedicine, Anhui Medical University (No. CH1059). All patients signed informed written consent before enrollment in this study.

\section{Laboratory Biochemical Examination}

All subjects were asked to do an overnight fast Venous blood samples were obtained from all study participants and analyzed by Biaojia Biotechnology Laboratory in Shenzhen, China. Lipids (including total cholesterol (TC, mmol/L), triglycerides ( $\mathrm{TG}, \mathrm{mmol} / \mathrm{L})$, highdensity lipoprotein-cholesterol (HDL-C, mmol/L), lowdensity lipoprotein cholesterol (LDL-C, mmol/L)), ${ }^{12,13}$ estimated glomerular filtration rate (eGFR, $\mathrm{mL} / \mathrm{min} /$ $1.73 \mathrm{~m}^{2}$ ), fasting blood glucose (FBG, mmol/L) and homocysteine $(\mathrm{Hcy}, \mu \mathrm{mol} / \mathrm{L})$ were measured using automatic clinical analyzers (Beckman Coulter, USA) and the laboratory staff were blind to the research protocol.

\section{Measurement of BMI}

The height and weight of the subjects were measured by trained staff using standardized equipment in accordance with standard operation procedure. BMI $=$ Weight $(\mathrm{kg}) /$ Height $(\mathrm{m})^{2}$.

\section{Measurement of $A B I$ and Definition of PAD}

The ABI of each lower limb was calculated by dividing the systolic pressure of the ankle artery of the corresponding lower limb by the systolic pressure of the brachial artery. Subjects rested quietly in a warm room for more than 10 minutes and fully exposed their upper limbs and ankles. Trained technicians used the Omron Colin BP203RPE III device (Omron Health Care, Kyoto, Japan) to simultaneously measure bilateral brachial and ankle arterial systolic pressures in supine subjects. And the software automatically calculates the bilateral ABI data according to the above calculation formula. All measurements were conducted in accordance with strict standard protocols. PAD was defined as an $\mathrm{ABI} \leq 0.9$ in either lower limb. ${ }^{14}$ Subjects with ABI $>1.4$ were excluded because of abnormal elevation of ABI may due to calcification of the arterial wall. ${ }^{15}$

\section{Other Variables}

Variables included age (years), sex, systolic blood pressure (SBP, mmHg) and diastolic blood pressure (DBP, $\mathrm{mmHg}$ ) measured by electronic sphygmomanometers after the subjects had rested for 10 minutes. Qualified researchers were trained to collect information by using standardized questionnaires, including smoking status (never, former, current), alcohol consumption (never, former, current), antihypertensive drugs (yes or no), the history of comorbid diseases including diabetes mellitus (yes or no), stroke (yes or no), and coronary heart disease (yes or no).

\section{Statistical Analysis}

Normally distributed variables were presented as mean \pm standard deviation (SD); for non-normally distributed data the median and inter-quartile range (IQR) are given, and categorical variables as percentage (\%). Population characteristics were described according to BMI classify. To reduce redundancy, variance inflation factors (VIF) were used to assess collinearity between independent variables before our data analysis, with a variable having VIF $>5$ considered collinear with other variables. In comparison, LDL-C (VIF=5.9) had to be excluded from the next analysis because of its collinearity to other variables. The dose-response relationship between BMI and the risk of PAD was estimated using generalized additive regression model and smoothing curve (penalized spline method) with adjustment for age, sex, systolic and diastolic blood 
pressure, pulse rate, smoking status, alcohol consumption, TC, TG, HDL-C, eGFR, Hcy, antihypertensive drugs, diabetes mellitus, stroke, coronary heart disease. If nonlinear was detected, threshold effect analysis was used for inflection points of BMI by using segmented regression model, LRT test and bootstrap resampling method. Multivariate logistic regression was used to analyze the relationship between BMI and the risk of PAD around threshold value. $P$ value for interaction was used to compare whether there was a significant difference in the correlation between BMI and the risk of PAD before and after inflection point. In addition, possible modifications of the association between BMI and PAD were assessed for variables including sex, age, blood pressure controlled, pulse rate, Hcy, lipids profile, smoking status, history of diabetes mellitus and stroke.

All analyses in this study with $\mathrm{P}$ values $<0.05$ (twotailed) were considered statistically significant. All analyses were statistically analyzed by EnpowerStats (www. empowerstats.com; X\&Y Solutions, Inc., Boston, MA) and R statistical software (http://www.r-project.org).

\section{Results}

\section{Baseline Characteristics of Participants}

As shown in Table 1, a total of 10,896 hypertensive patients with a mean age of $63.9 \pm 9.3$ years were included in this study. The prevalence of PAD was $3.2 \%$, the mean BMI was $23.6 \pm 3.6 \mathrm{~kg} / \mathrm{m}^{2}$, and $47.1 \%$ were male. BMI was stratified to four groups: underweight (BMI $<18.5 \mathrm{~kg} /$ $\left.\mathrm{m}^{2}\right)$, normal $\left(\mathrm{BMI} \geq 18.5, \quad<25 \mathrm{~kg} / \mathrm{m}^{2}\right)$, overweight $\left(\mathrm{BMI} \geq 25,>30 \mathrm{~kg} / \mathrm{m}^{2}\right)$ and obesity $\left(\mathrm{BMI} \geq 30 \mathrm{~kg} / \mathrm{m}^{2}\right)$ to describe demographic characteristics. The underweight of participants accounted for $6.3 \%$ of the total population, and obesity was only $4.2 \%$. The prevalence of PAD in underweight was the highest $(6.7 \%)$ and followed by obesity (4.4\%), while overweight was only $2.3 \%$. Compared with the other three groups, underweight participants were older, with higher tHcy, HDL-C, current smoking rate, and lower TC, TG, eGFR, the prevalence of diabetes mellitus and the use of the antihypertensive drug.

\section{Association Between BMI and PAD}

As shown in Figure 1, the relationship between BMI and the prevalence of PAD showed a U-shaped curve, and threshold saturation effect analysis showed that BMI value with the lowest risk of PAD was estimated to be
$25.7 \mathrm{~kg} / \mathrm{m}^{2}$. We stratified BMI by $25.7 \mathrm{~kg} / \mathrm{m}^{2}$ and used logistic regression analysis models (Table 2). Per SD increment $\left(3.6 \mathrm{~kg} / \mathrm{m}^{2}\right)$ on the left side of the threshold $\left(\mathrm{BMI}<25.7 \mathrm{~kg} / \mathrm{m}^{2}\right)$, BMI was associated with a $27 \%$ decrease in the risk of PAD [adjusted odds ratio (OR), $0.73 ; 95 \%$ confidence interval $(\mathrm{CI}) 0.60,0.89 ; \mathrm{P}=0.002$ ]; however, BMI was significantly positively associated with the risk of PAD (adjusted OR, 1.52; 95\% CI 1.52, 1.93; $\mathrm{P}=0.001$ ) in those with $\mathrm{BMI} \geq 25.7 \mathrm{~kg} / \mathrm{m}^{2}$. Further adjusted lipid-lowering drugs as a sensitivity analysis, no change to the result suggested that the result was stable (Supplemental Table 1).

\section{Stratified Analyses by Potential Effect Covariables}

None of other covariables, including sex (male vs female), age ( $<65$ vs $\geq 65$ years), blood pressure controlled [yes vs no (yes: $\mathrm{SBP}<140 \mathrm{mmHg}$ and $\mathrm{DBP}<90 \mathrm{mmHg}$; otherwise no)], pulse rate ( $<75 \mathrm{vs} \geq 75 \mathrm{bmp}$ ), smoking status (never vs former vs current), total Hcy $(<15$ vs $\geq 15 \mu \mathrm{mol} / \mathrm{L})$, total cholesterol ( $<5.2$ vs. $\geq 5.2 \mathrm{mmol} / \mathrm{L})$, HDL-C[abnormal vs normal (normal: male HDL-C $\geq 1.04 \mathrm{mmol} / \mathrm{L}$, female HDL$\mathrm{C} \geq 1.3 \mathrm{mmol} / \mathrm{L}$; abnormal: male HDL-C $<1.04 \mathrm{mmol} / \mathrm{L}$, female HDL-C $<1.3 \mathrm{mmol} / \mathrm{L}$ )], diabetes mellitus (yes vs no), stroke (yes vs no) significantly modified the association between $\mathrm{BMI}$ and the risk of PAD, whether in the hypertensive population with $\mathrm{BMI}<25.7 \mathrm{~kg} / \mathrm{m}^{2}$ or $\mathrm{BMI} \geq 25.7 \mathrm{~kg} / \mathrm{m}^{2}$ (All stratified P-interactions were $>0.05$ ) (Figure 2).

\section{Discussion}

In our analysis of this community-based hypertension registry study in China, we noted a U-shaped relationship between BMI and risk of PAD. The BMI value with lowest risk of PAD was estimated to be $25.7 \mathrm{~kg} / \mathrm{m}^{2}$.

A number of studies have reported the relationship between BMI and the risk of PAD. However, the association between BMI and PAD risk was not consistent. Epidemiological studies more than two decades ago reported a positive association between BMI and intermittent claudication in middle-aged males in Israel. $^{16}$ However, many population studies after adjusting for the relevant covariates fail to support the significant association between BMI and the prevalence of PAD. ${ }^{4,17}$ In addition, the San Diego study reported an independent and significantly inverse association between BMI and prevalence of PAD (OR: 0.88) in multi-ethnic population. $^{18}$ Studies on the diabetic population in 
Table I Population Characteristics of Stratified by Body Mass Index

\begin{tabular}{|c|c|c|c|c|c|c|}
\hline \multirow[t]{2}{*}{ Characteristics } & \multirow[t]{2}{*}{ Total } & \multicolumn{4}{|c|}{ Body Mass Index $\left(\mathrm{kg} / \mathrm{m}^{2}\right)$} & \multirow[t]{2}{*}{ P-value } \\
\hline & & $\begin{array}{c}\text { Underweight: } \\
\quad<18.5\end{array}$ & $\begin{array}{c}\text { Normal: } \geq 18.5, \\
<25\end{array}$ & $\begin{array}{c}\text { Overweight: } \geq 25, \\
<30\end{array}$ & $\begin{array}{c}\text { Obesity: } \\
\geq \mathbf{3 0}\end{array}$ & \\
\hline $\mathrm{N}$ & 10,896 & 691 & 6594 & 3157 & 454 & \\
\hline Age, y & $63.9 \pm 9.3$ & $70.7 \pm 8.3$ & $64.9 \pm 8.8$ & $61.0 \pm 9.1$ & $58.7 \pm 9.4$ & $<0.001$ \\
\hline BMI, $\mathrm{kg} / \mathrm{m}^{2}$ & $23.6 \pm 3.6$ & $17.4 \pm 0.9$ & $22.1 \pm 1.7$ & $26.8 \pm 1.3$ & $32.2 \pm 3.0$ & $<0.001$ \\
\hline $\mathrm{SBP}, \mathrm{mmHg}$ & $148.5 \pm 17.8$ & $147.4 \pm 20.0$ & $148.7 \pm 17.9$ & $148.1 \pm 17.0$ & $149.5 \pm 17.4$ & 0.071 \\
\hline $\mathrm{DBP}, \mathrm{mmHg}$ & $89.0 \pm 10.7$ & $83.6 \pm 11.6$ & $88.4 \pm 10.5$ & $91.0 \pm 10.4$ & $92.3 \pm 10.9$ & $<0.001$ \\
\hline Pulse rate, bpm & $76.3 \pm 14.2$ & $77.1 \pm 15.1$ & $75.8 \pm 14.4$ & $77.0 \pm 13.7$ & $78.3 \pm 11.8$ & $<0.001$ \\
\hline PAD, N(\%) & $35 I(3.2)$ & $46(6.7)$ & $212(3.2)$ & $73(2.3)$ & $20(4.4)$ & $<0.001$ \\
\hline \multicolumn{7}{|l|}{ Lab Examination } \\
\hline Homocysteine, $\mu \mathrm{mol} / \mathrm{L}$ & $18.0 \pm 11.0$ & $19.3 \pm 10.8$ & $18.1 \pm 11.0$ & $17.5 \pm 10.9$ & $17.7 \pm 13.0$ & $<0.001$ \\
\hline Fasting blood glucose, $\mathrm{mmol} / \mathrm{L}$ & $6.2 \pm 1.6$ & $5.8 \pm 1.1$ & $6.1 \pm 1.5$ & $6.4 \pm 1.9$ & $6.5 \pm 1.8$ & $<0.001$ \\
\hline Total cholesterol, mmol/L & $5.1 \pm 1.1$ & $4.9 \pm 1.1$ & $5.1 \pm 1.1$ & $5.2 \pm 1.1$ & $5.2 \pm 1.1$ & $<0.001$ \\
\hline Triglyceride, $\mathrm{mmol} / \mathrm{L}$ & $1.4(1.0-2.1)$ & $1.0(0.8-1.3)$ & $1.4(1.0-1.9)$ & $1.8(1.3-2.6)$ & $1.8(1.3-2.6)$ & $<0.001$ \\
\hline HDL-C, mmol/L & $1.6 \pm 0.4$ & $1.8 \pm 0.5$ & $1.6 \pm 0.4$ & $1.5 \pm 0.4$ & $1.5 \pm 0.4$ & $<0.001$ \\
\hline LDL-C, mmol/L & $3.0 \pm 0.8$ & $2.6 \pm 0.7$ & $2.9 \pm 0.8$ & $3.1 \pm 0.8$ & $3.1 \pm 0.8$ & $<0.001$ \\
\hline eGFR, $\mathrm{mL} / \mathrm{min} / 1.73 \mathrm{~m}^{2}$ & $88.7 \pm 20.4$ & $80.9 \pm 21.7$ & $88.3 \pm 20.0$ & $90.5 \pm 20.4$ & $93.4 \pm 20.6$ & $<0.001$ \\
\hline Sex, N(\%) & & & & & & $<0.001$ \\
\hline male & $5127(47.1)$ & $359(52.0)$ & $3193(48.4)$ & 1402 (44.4) & $173(38.1)$ & \\
\hline female & $5769(52.9)$ & $332(48.0)$ & $340 I(51.6)$ & $1755(55.6)$ & $28 I(6 I .9)$ & \\
\hline Smoking status, $\mathrm{N}(\%)$ & & & & & & $<0.001$ \\
\hline Never & $6277(57.6)$ & 317 (45.9) & $3699(56.1)$ & $1956(62.0)$ & $305(67.3)$ & \\
\hline Former & $175 \mid(16.1)$ & $114(16.5)$ & $1052(16.0)$ & $526(16.7)$ & $59(13.0)$ & \\
\hline Current & $2867(26.3)$ & $260(37.6)$ & $1843(27.9)$ & $675(21.4)$ & $89(19.6)$ & \\
\hline Alcohol consumption, $\mathrm{N}(\%)$ & & & & & & 0.013 \\
\hline Never & $6842(62.8)$ & $438(63.4)$ & $4075(61.8)$ & $2011(63.7)$ & $318(70.2)$ & \\
\hline Former & I584 (I4.5) & $98(14.2)$ & $974(14.8)$ & $452(14.3)$ & $60(13.2)$ & \\
\hline Current & $2468(22.7)$ & $155(22.4)$ & $1544(23.4)$ & $694(22.0)$ & $75(16.6)$ & \\
\hline Diabetes mellitus, $\mathrm{N}(\%)$ & $1238(11.4)$ & $23(3.3)$ & $642(9.7)$ & $474(15.0)$ & $99(21.8)$ & $<0.001$ \\
\hline Stroke, N(\%) & $706(6.5)$ & $43(6.2)$ & $44 \mid(6.7)$ & $205(6.5)$ & $17(3.7)$ & 0.104 \\
\hline $\mathrm{CHD}, \mathrm{N}(\%)$ & $552(5.1)$ & $46(6.7)$ & $336(5.1)$ & $146(4.6)$ & $24(5.3)$ & 0.174 \\
\hline Antihypertensive drugs, $\mathrm{N}(\%)$ & $7154(65.7)$ & $406(58.8)$ & $4272(64.8)$ & $2162(68.5)$ & $314(69.3)$ & $<0.001$ \\
\hline Lipid-lowering drugs, $N(\%)$ & $381(3.5)$ & $7(1.0)$ & $199(3.0)$ & $154(4.9)$ & $21(4.6)$ & $<0.001$ \\
\hline
\end{tabular}

Notes: Values are $\mathrm{N}(\%)$ or mean $\pm \mathrm{SD}$, except triglyceride presented as the median (IQR).

Abbreviations: BMI, body mass index, SBP, systolic blood pressure; DBP, diastolic blood pressure; PAD, peripheral vascular disease; HDL-C, high-density lipid cholesterol; FBG, fasting blood glucose; tHcy, total Homocysteine; eGFR, estimated glomerular filtration rate; CHD, coronary heart disease; IQR, inter-quartile range.

Taiwan showed that compared with diabetic patients without PAD, the BMI of patients with PAD was lower $(23.5$ \pm 3.2 vs. $\left.24.8 \pm 3.5 \mathrm{~kg} / \mathrm{m}^{2}, \mathrm{P}<0.005\right)$. Heffron et al who gathered data from more than 20,000 sites $(n=3,250,350)$ in the United States from 2003 to 2008, recently reported $\mathrm{BMI}$ and the prevalence of PAD in females showed a J-shaped nonlinear relationship; a significant positive correlation between obesity and PAD in females, while only a slight positive correlation between obesity (BMI $\geq$ $\left.40 \mathrm{~kg} / \mathrm{m}^{2}\right)$ and PAD in males $(\mathrm{OR}=2.98 \mathrm{vs} 1.37) .{ }^{9}$ Stepwise logistic regression analysis showed that the association between BMI and PAD was inverse. ${ }^{19}$ 


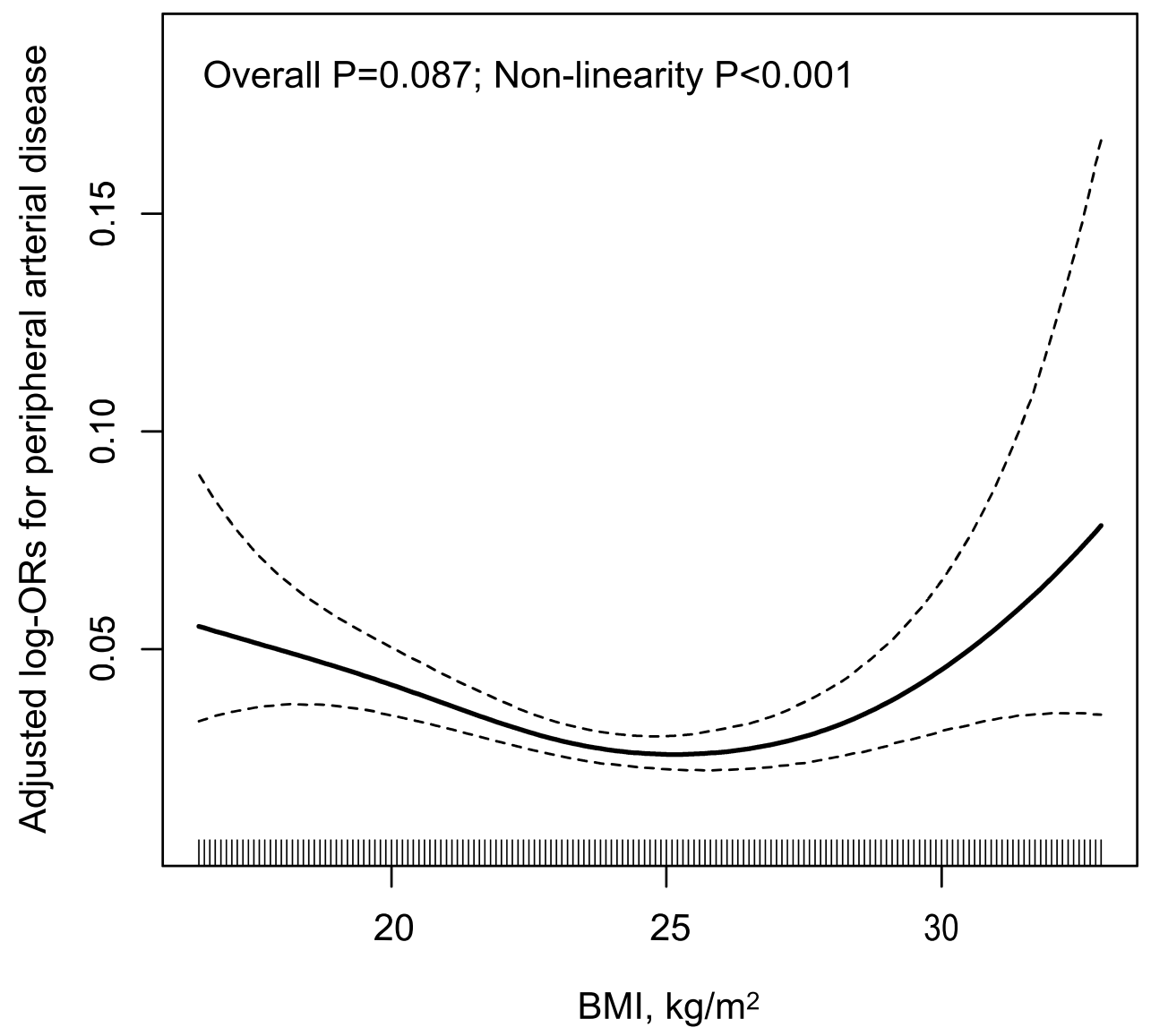

Figure I Smoothing curve of association between BMI and the risk of PAD. Adjusted for: age, sex, systolic and diastolic blood pressure, pulse rate, smoking status, alcohol consumption, total cholesterol, triglyceride, high density lipoprotein cholesterol, fasting blood glucose, estimated glomerular filtration rate, total homocysteine, antihypertensive drugs, diabetes mellitus, stroke, coronary heart disease.

To our knowledge, the U-shaped relationship between BMI and the risk of PAD shown in our study was the first reported in Chinese hypertensive population. Different from the very large sample population studies ${ }^{9}$ in the United States, where participants were nearly $30 \%$ obese and $3.4 \%$ underweight, as well as study of the prevalence of PAD in African, ${ }^{10}$ where obesity was only $4.5 \%, 34.1 \%$ underweight, we were $6.3 \%$ (691) underweight and only $4.2 \%$ (454) obesity, nearly $90 \%$ of the population was normal BMI and overweight. Over a third of the study population was underweight. A U-shaped relationship between BMI and the risk of PAD was observed. Compare to the subjects with normal BMI, underweight and obesity were statistically significant association with

Table 2 Association of BMI and the Risk of PAD Stratified by BMI Threshold

\begin{tabular}{|c|c|c|c|c|c|c|c|c|}
\hline $\begin{array}{l}\text { BMI, kg/m² (per SD } \\
\text { Increment) }\end{array}$ & $\mathbf{N}$ & $\begin{array}{c}\text { Events } \\
(\%)\end{array}$ & $\begin{array}{c}\text { Crude Model OR } \\
\qquad(95 \% \mathrm{Cl})\end{array}$ & $P$ value & $\begin{array}{c}\text { Model I OR } \\
(95 \% \mathrm{Cl})\end{array}$ & $P$ value & $\begin{array}{c}\text { Model } 2 \text { OR } \\
(95 \% \mathrm{CI})\end{array}$ & $P$ value \\
\hline Total participants & 10896 & $35 I(3.2)$ & $0.75(0.67,0.84)$ & $<0.001$ & $1.02(0.91,1.15)$ & 0.689 & $0.96(0.85,1.10)$ & 0.559 \\
\hline$<25.7$ & 8027 & $278(3.5)$ & $0.55(0.47,0.66)$ & $<0.001$ & $0.83(0.69,1.00)$ & 0.048 & $0.73(0.60,0.89)$ & 0.002 \\
\hline$\geq 25.7$ & 2869 & $73(2.5)$ & $1.31(1.04,1.65)$ & 0.020 & $1.38(1.10,1.73)$ & 0.006 & $1.52(1.20,1.93)$ & 0.001 \\
\hline$P$ for interaction & & & & $<0.001$ & & 0.001 & & $<0.001$ \\
\hline Log Likelihood Ratio Tests & & & & & & & & 0.002 \\
\hline
\end{tabular}

Notes: Crude model adjust for none; Model I adjust for age, sex, diabetes mellitus, smoking status; Model 2 adjust for: age, sex, systolic and diastolic blood pressure, pulse rate, smoking status, alcohol consumption, total cholesterol, triglyceride, high density lipoprotein cholesterol, estimated glomerular filtration rate, total homocysteine, antihypertensive drugs, diabetes mellitus, stroke, coronary heart disease.

Abbreviations: $\mathrm{Cl}$, confidence interval; BMI, Body Mass Index; PAD, peripheral arterial disease. 


Variables
Sex
male
female
Age, ys
$<65$
$\geq 65$
Blood pressure controlled
yes
no
Homocysteine, $\mu \mathrm{mol} / \mathrm{L}$
$<15$
$\geq 15$
Total cholesterol, $\mathrm{mmol} / \mathrm{L}$
$<5.2$
$\geq 5.2$
HDL-C, mmol/L
normal
abnormal
eGFR, ml/(min ${ }^{*} 1.73 \mathrm{~m}^{2}$ )
$<90$
$\geq 90$
Smoking status
Never
Former
Current
Diabetes mellitus
No
Yes
Stroke
No
Yes

$\begin{array}{cc}\mathbf{N} & \text { Events No. (\%) } \\ & \\ 3892 & 165(4.2) \\ 4135 & 113(2.7) \\ & \\ 3492 & 49(1.4) \\ 4535 & 229(5.0) \\ & \\ 1961 & 69(3.5) \\ 6066 & 209(3.4) \\ & \\ 3798 & 79(2.1) \\ 4224 & 198(4.7) \\ & \\ 4425 & 159(3.6) \\ 3597 & 118(3.3) \\ & \\ 6878 & 235(3.4) \\ 1144 & 42(3.7) \\ & \\ 3539 & 187(5.3) \\ 4483 & 90(2.0) \\ 4464 & 108(2.4) \\ 1296 & 43(3.3) \\ 2267 & 127(5.6) \\ 7270 & 255(3.5) \\ 757 & 23(3.0) \\ 7502 & 241(3.2) \\ 525 & 37(7.0) \\ & \\ & \end{array}$

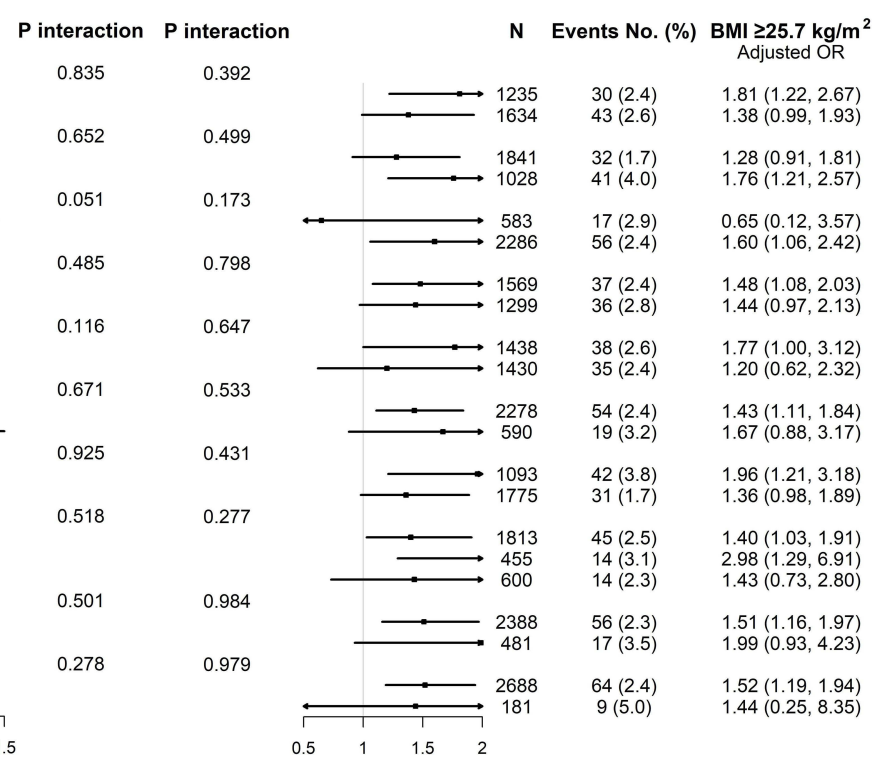

Figure 2 Subgroup analyses on the association between BMI and the risk of PAD. Adjusted for: age, sex, systolic and diastolic blood pressure, pulse rate, smoking status, alcohol consumption, total cholesterol, triglyceride, high density lipoprotein cholesterol, fasting blood glucose, estimated glomerular filtration rate, total homocysteine, antihypertensive drugs, diabetes mellitus, stroke, coronary heart disease, except for the stratifying variable.

the risk of PAD (OR, 2.09; 95\% CI 1.35, 3.22; $\mathrm{P}=0.0009$; OR, $1.90 ; 95 \%$ CI $1.04,3.23 ; \mathrm{P}=0.0336$ ), but not overweight $(\mathrm{OR}, 1.56 ; 95 \%$ CI $0.70,2.51 ; \mathrm{P}=0.7342) .{ }^{10}$ However, Heffron et al found a "J-shaped" relationship between BMI and PAD only in females, not in males, which may be due to the height and weight data used in this study for self-reporting of participants. Self-reported data may lead to personal BMI classification appear serious mistakes, ${ }^{20}$ difficult to correct the mistakes, ${ }^{21}$ especially in the stratified analysis according to gender. ${ }^{22}$ Thus, self-report bias may have contributed to the fact that this study found a "J-shaped" relationship between BMI and PAD risk only in females, and not in males.

At present, few studies have elaborated on the possible mechanism of the correlation between BMI and PAD. A cross-sectional study of hemodialysis patients reported a lower prevalence of atherosclerosis and lower levels of inflammation (CRP) in patients with normal BMI and overweight compared with those with underweight and obesity. ${ }^{23}$ Lower levels of inflammation and atherosclerosis may be associated with the lowest risk of PAD in this population (normal BMI and overweight).

Not only that, there have been also many reports on the U-shaped relationship between BMI and cardiovascular disease and death. A meta-analysis of 97 studies showed that obesity (all grades) and grades 2 and 3 obesity were significantly associated with all-cause mortality relative to normal BMI. However, overweight was associated with a significant reduction in all-cause mortality. ${ }^{22}$ Among more than 1 million East Asian populations in the Asia Cohort Consortium BMI Project, including Chinese, Japanese, and Korean, the Cox proportional hazard regression model was used to analyze the relationship between BMI and mortality risk, which showed that the population with BMI between 22.6 and 27.5 had the lowest mortality risk. ${ }^{24}$ Based on this, we speculate that the "U-shaped" relationship between BMI and peripheral atherosclerosis may, on one hand, explain the causes of the lowest cardiovascular disease risk and all-cause mortality in normal BMI/overweight.

\section{Limitations and Future Directions}

Nonetheless, these results must be interpreted with caution, and a number of limitations should be borne in mind. First, subjects in our analysis were middle-aged and elderly patients with hypertension. The U-shaped relationship between BMI and the risk of PAD was not necessarily applicable to the general population, but as an independent risk factor for PAD, exploring the relationship between BMI and the risk of PAD in the hypertensive population can serve the high-risk population more precisely. In addition, the association between BMI and the risk of PAD was still controversial. By design, our study was a crosssectional study and cannot study the chronology of BMI and PAD. There might be a reverse causal relationship. The weight change caused by the disease may distort the 
relationship between $\mathrm{BMI}$ and $\mathrm{PAD}$. In the future, large prospective cohort studies on PAD were urgently needed. Final, the obesity rate in our study was low. It has no enough power to assess the relationship between different degrees of obesity or morbid obesity and the risk of PAD. However, our study reflects the real situation of hypertension population in Chinese hypertension, and the results obtained were more suitable for the application of hypertension in middle-aged and elderly people in China.

\section{Conclusions}

Our study reported the prevalence of PAD was $3.2 \%$. The U-shaped association between BMI and the risk of PAD was found in Chinese middle-aged and elderly patients with hypertension. BMI with the lowest risk of PAD was estimated to be $25.7 \mathrm{~kg} / \mathrm{m}^{2}$ in our study.

\section{Data Sharing Statement}

The raw data used to support the findings of this study are available from the corresponding author upon request.

\section{Acknowledgments}

We thank all investigators and participants in the China Hypertension Registry, the parent study, who made this report possible.

\section{Funding}

This work was funded by the Jiangxi Outstanding Person Foundation (grant numbers[20192BCBL23024]). Key R\&D Projects, Jiangxi [20203BBGL73173]. 5511 Science and Technology Innovation Talent Project of Jiangxi Province (CN) (Grant numbers [20165BCD41005]). National Key R\&D Program of China (grant numbers [2018YFC1312902]). The National Natural Science Foundation of China (CN) (Grant numbers [81760049] and [81960074]). Health Commission of Jiangxi Province Foundation [202130440]. Key projects of Jiangxi Provincial Education Department (grant numbers [GJJ170013]).

\section{Disclosure}

Dr Xiaoshu Cheng reports grants from Jiangxi Outstanding Person Foundation, grants from Key R\&D Projects, Jiangxi, grants from 5511 Science and Technology Innovation Talent Project of Jiangxi Province $(\mathrm{CN})$, grants from National Key R\&D Program of China, grants from The National Natural Science Foundation of China (CN), grants from Health Commission of Jiangxi
Province Foundation, grants from Key projects of Jiangxi Provincial Education Department, during the conduct of the study. The authors declare that they have no conflict of interest.

\section{References}

1. Lozano R, Naghavi M, Foreman K, et al. Global and regional mortality from 235 causes of death for 20 age groups in 1990 and 2010: a systematic analysis for the Global Burden of Disease Study 2010. Lancet. 2012;380(9859):2095-2128. doi:10.1016/S0140-6736(12) 61728-0

2. Savji N, Rockman CB, Skolnick AH, et al. Association between advanced age and vascular disease in different arterial territories: a population database of over 3.6 million subjects. $\mathrm{J} \mathrm{Am} \mathrm{Coll}$ Cardiol. 2013;61(16):1736-1743. doi:10.1016/j.jacc.2013.01.054

3. Criqui MH, Aboyans V. Epidemiology of peripheral artery disease. Circ Res. 2015;116(9):1509-1526. doi:10.1161/CIRCRESAHA.1 16.303849

4. Allison MA, Criqui MH, McClelland RL, et al. The effect of novel cardiovascular risk factors on the ethnic-specific odds for peripheral arterial disease in the Multi-Ethnic Study of Atherosclerosis (MESA). $J$ Am Coll Cardiol. 2006;48(6):1190-1197. doi:10.1016/j. jacc.2006.05.049

5. Vitalis A, Lip GY, Kay M, Vohra RK, Shantsila A. Ethnic differences in the prevalence of peripheral arterial disease: a systematic review and meta-analysis. Expert Rev Cardiovasc Ther. 2017;15(4):327-338. doi:10.1080/14779072.2017.1305890

6. Huang Y, Xu M, Xie L, et al. Obesity and peripheral arterial disease: a Mendelian Randomization analysis. Atherosclerosis. 2016;247:218-224. doi:10.1016/j.atherosclerosis.2015.12.034

7. Ix JH, Biggs ML, Kizer JR, et al. Association of body mass index with peripheral arterial disease in older adults: the Cardiovascular Health Study. Am J Epidemiol. 2011;174(9):1036-1043. doi:10.1093/ aje/kwr228

8. Golledge J, Leicht A, Crowther RG, Clancy P, Spinks WL, Quigley F. Association of obesity and metabolic syndrome with the severity and outcome of intermittent claudication. J Vasc Surg. 2007;45(1):40-46. doi:10.1016/j.jvs.2006.09.006

9. Heffron SP, Dwivedi A, Rockman CB, et al. Body mass index and peripheral artery disease. Atherosclerosis. 2020;292:31-36. doi:10.1016/j.atherosclerosis.2019.10.017

10. Desormais I, Aboyans V, Guerchet M, et al. Body mass index and peripheral arterial disease, a "U-shaped" relationship in elderly African population - the EPIDEMCA study. Vasa. 2020;49 (1):50-56. doi:10.1024/0301-1526/a000825

11. Yu Y, Hu L, Huang X, Zhou W, Bao H, Cheng X. BMI modifies the association between serum HDL cholesterol and stroke in a hypertensive population without atrial fibrillation. J Endocrinol Invest. 2020

12. Cicero AFG, Fogacci F, Giovannini M, et al. Interaction between low-density lipoprotein-cholesterolaemia, serum uric level and incident hypertension: data from the Brisighella Heart Study. $J$ Hypertens. 2019;37(4):728-731. doi:10.1097/HJH.0000000 000001927

13. Cicero AFG, Fogacci F, Tocci G, et al. Awareness of major cardiovascular risk factors and its relationship with markers of vascular aging: data from the Brisighella Heart Study. Nutr Metab Cardiovasc Dis. 2020;30(6):907-914. doi:10.1016/j.numecd.2020.03.005

14. Guirguis-Blake JM, Evans CV, Redmond N, Lin JS. Screening for peripheral artery disease using the ankle-brachial index: updated evidence report and systematic review for the US preventive services task force. JAMA. 2018;320(2):184-196. doi:10.1001/jama.20 18.4250 
15. Wassel CL, Berardi C, Pankow JS, et al. Soluble P-selectin predicts lower extremity peripheral artery disease incidence and change in the ankle brachial index: the Multi-Ethnic Study of Atherosclerosis (MESA). Atherosclerosis. 2015;239(2):405-411. doi:10.1016/j. atherosclerosis.2015.01.022

16. Bowlin SJ, Medalie JH, Flocke SA, Zyzanski SJ, Goldbourt U. Epidemiology of intermittent claudication in middle-aged men. $\mathrm{Am}$ $J$ Epidemiol. 1994;140(5):418-430. doi:10.1093/oxfordjournals.aje. a117264

17. Murabito JM, Evans JC, Nieto K, Larson MG, Levy D, Wilson PW. Prevalence and clinical correlates of peripheral arterial disease in the Framingham Offspring Study. Am Heart J. 2002;143(6):961-965. doi:10.1067/mhj.2002.122871

18. Criqui MH, Vargas V, Denenberg JO, et al. Ethnicity and peripheral arterial disease: the San Diego Population Study. Circulation. 2005;112 (17):2703-2707. doi:10.1161/CIRCULATIONAHA.105.546507

19. Tseng $\mathrm{CH}$. Prevalence and risk factors of peripheral arterial obstructive disease in Taiwanese type 2 diabetic patients. Angiology. 2003;54 (3):331-338. doi:10.1177/000331970305400309
20. Spencer EA, Appleby PN, Davey GK, Key TJ. Validity of self-reported height and weight in 4808 EPIC-Oxford participants. Public Health Nutr. 2002;5(4):561-565. doi:10.1079/PHN2001322

21. Plankey MW, Stevens J, Flegal KM, Rust PF. Prediction equations do not eliminate systematic error in self-reported body mass index. Obes Res. 1997;5(4):308-314. doi:10.1002/j.1550-8528.1997.tb00556.x

22. Flegal KM, Kit BK, Orpana H, Graubard BI. Association of all-cause mortality with overweight and obesity using standard body mass index categories: a systematic review and meta-analysis. JAMA. 2013;309(1):71-82. doi:10.1001/jama.2012.113905

23. Kahraman S, Yilmaz R, Akinci D, et al. U-shaped association of body mass index with inflammation and atherosclerosis in hemodialysis patients. J Ren Nutr. 2005;15(4):377-386. doi:10.1053/j. jrn.2005.07.004

24. Zheng W, McLerran DF, Rolland B, et al. Association between body-mass index and risk of death in more than 1 million Asians. N Engl J Med. 2011;364(8):719-729. doi:10.1056/NEJMoa1010679

\section{Publish your work in this journal}

The International Journal of General Medicine is an international, peer-reviewed open-access journal that focuses on general and internal medicine, pathogenesis, epidemiology, diagnosis, monitoring and treatment protocols. The journal is characterized by the rapid reporting of reviews, original research and clinical studies across all disease areas. The manuscript management system is completely online and includes a very quick and fair peer-review system, which is all easy to use. Visit http://www.dovepress.com/ testimonials.php to read real quotes from published authors. 